Canadian University Music Review

Canadian University Music Review

Revue de musique des universités canadiennes

\title{
Une nouvelle découverte à Montréal dans la bibliothèque musicale de Jean Girard : des œuvres vocales rares de Lebègue, de Bacilly et de Du Mont
}

\section{Élisabeth Gallat-Morin}

Volume 17, numéro 2, 1997

URI : https://id.erudit.org/iderudit/1014784ar

DOI : https://doi.org/10.7202/1014784ar

Aller au sommaire du numéro

\section{Éditeur(s)}

Canadian University Music Society / Société de musique des universités canadiennes

\section{ISSN}

0710-0353 (imprimé)

2291-2436 (numérique)

Découvrir la revue

\section{Citer cet article}

Gallat-Morin, É. (1997). Une nouvelle découverte à Montréal dans la bibliothèque musicale de Jean Girard : des œuvres vocales rares de Lebègue, de Bacilly et de Du Mont. Canadian University Music Review / Revue de musique des universités canadiennes, 17(2), 17-29. https://doi.org/10.7202/1014784ar
Résumé de l'article

Un recueil donné à la Bibliothèque nationale du Québec (Montréal) en 1993 porte les signatures de François Vachon de Belmont, supérieur des Sulpiciens de Montréal de 1701 à 1732, et de Jean Girard, à qui appartenait le Livre d'orgue de Montréal. Les quatre volumes renferment les Motets (1687) de M. Noël (pseudonyme de Lebègue), les Airs spirituels de "différents autheurs » (1697), édition " pirate » d'œuvres de Baccilly, et les Airs à quatre parties (1663) de Du Mont. Très rares, voire uniques, ces œuvres complètent la bibliothèque musicale de Jean Girard et ajoutent à notre connaissance du répertoire musical interprété à Ville-Marie au XVIII ${ }^{\mathrm{e}}$ siècle.
All Rights Reserved ( C Canadian University Music Society / Société de musique des universités canadiennes, 1997
Ce document est protégé par la loi sur le droit d'auteur. L'utilisation des services d'Érudit (y compris la reproduction) est assujettie à sa politique d'utilisation que vous pouvez consulter en ligne.

https://apropos.erudit.org/fr/usagers/politique-dutilisation/ 


\title{
UNE NOUVELLE DÉCOUVERTE À MONTRÉAL DANS LA BIBLIOTHÈQUE MUSICALE DE JEAN GIRARD : DES CEUVRES VOCALES RARES DE LEBÈGUE, DE BACILLY ET DE DU MONT
}

\author{
Élisabeth Gallat-Morin
}

Nous pensions tout savoir sur l'activité musicale du clerc sulpicien Jean Girard (1696-1765). C'est grâce à lui qu'on a préservé le plus volumineux manuscrit de pièces d'orgue de l'époque de Louis XIV à avoir survécu, le Livre d'orgue de Montréal. Nous savons aussi qu'il a œuvré pendant 40 ans au centre de la vie montréalaise dans les fonctions modestes, mais combien importantes, de maître d'école et d'organiste responsable de la musique liturgique à la paroisse Notre-Dame. Outre le manuscrit de pièces d'orgue, il avait apporté de France deux ouvrages de Guillaume-Gabriel Nivers : le Premier Livre d'orgue (tirage de 1667) et son Traité de la composition de musique publié la même année. Par ailleurs, nous avons retrouvé quelque 300 feuilles de plain-chant recopiées de la main de Girard : un recueil d'hymnes en latin, un autre de cantiques français notés en plain-chant, le propre de fêtes sulpiciennes ajouté à un antiphonaire appartenant à l'église Notre-Dame, l'office de la Visitation recopié à l'intention des sœurs de la Congrégation de Notre-Dame ${ }^{1}$. Il ne manquait pour compléter cette bibliothèque musicale que de la musique vocale autre que le plain-chant.

Voilà qu'un don à la Bibliothèque nationale du Québec à Montréal ${ }^{2}$ a fait surgir de l'oubli quatre petits recueils imprimés qui viennent étoffer la collection du musicien ecclésiastique : ils renferment des œuvres rares, des motets, des airs spirituels, ainsi que des airs sur la paraphrase des psaumes par Antoine Godeau, dont la musique se révélera être respectivement de Nicolas Lebègue,

1 Voir Élisabeth Gallat-Morin, Un manuscrit de musique française classique : étude critique et historique - Le Livre d'orgue de Montréal (Paris : Les Amateurs de livres/Klincsieck; Montréal : Les Presses de l'Université de Montréal, 1988); idem, Jean Girard, musicien en Nouvelle-France : Bourges, 1696-Montréal, 1765 (Sillery : Les éditions du Septentrion; Paris : Klincksieck, 1993). Voir aussi idem, "Un manuscrit de cantiques à Montréal (XVIII ${ }^{e}$ siècle) ", Revue de musique des universités canadiennes, $n^{\circ} 11 / 2$ (1991) : 68-93; idem, «Le Livre d'orgue de Montréal, aperçu d'un manuscrit inédit », Revue de musique des universités canadiennes, $\mathrm{n}^{\circ} 2$ (1981) : 1-38.

2Bibliothèque nationale du Québec, Montréal, Division des collections spéciales, cote RES AF 264. Nos remerciements vont à $M^{\mathrm{me}}$ Milada Vlach et à $M$. Denis Rivest qui nous ont signalé la présence de ce livre entré dans les collections à la suite d'un don à la fin de 1992. Le donateur n'a pas pu retracer la provenance du livre au-dela de son grand-père collectionneur, qui avait bien connu le bibliophile, l'abbe Anselme Verreau. 
de Bénigne de Bacilly et d'Henry Du Mont. Sur le méplat de la couverture avant, on peut lire les inscriptions manuscrites autographes : « Girard 1724 » et «Belmon ». En ce qui concerne Girard, l'inscription est la même que dans le Livre d'orgue de Montréal, la date de 1724 étant celle de son arrivée à Montréal $^{3}$. François Vachon de Belmont, pour sa part, était alors le supérieur des Sulpiciens de Montréal; auparavant, il avait été missionnaire au Fort de la Montagne où il accompagnait sur son luth les cantiques qu'il faisait chanter aux Indiens, en attendant l'arrivée de son orgue de Paris ${ }^{4}$. Accordant une grande importance à la musique et, une fois Supérieur, réclamant avec insistance l'envoi d'un maître d'école pouvant enseigner le chant, il a dû réserver un accueil particulièrement bienveillant au jeune maître d'école musicien de 28 ans, à qui il a remis les quatre recueils de musique vocale.

\section{Description physique et contenu}

Le volume de $16,5 \mathrm{~cm} \times 22 \mathrm{~cm}$ oblong possède une couverture de carton recouverte de toile assez grossière. Les coins sont usés, ainsi que la toile dans le haut et le bas de l'épine; la couverture arrière est brisée. Comme on le voit souvent, les pages ont été coupées en haut et en bas lors de la reliure. L'état du volume ainsi que de nombreux ajouts à l'encre (indications de ton, de cordes d'instrument, des paroles, deux chants avec musique, des chiffrages) témoignent du fait que le livre a réellement servi. Sur une douzaine de pages, une partie de l'anatomie des personnages, souvent nus, qui ornent les lettres au début des pièces, a été pudiquement barbouillée d'encre noires. Sur la feuille de garde arrière, quelqu'un a exercé ses talents de dessinateur : on y voit des tracés de lettres majuscules, comme des exercices d'écriture, mais aussi quatre essais de dessins de têtes d'enfants ou de chérubins faits d'une main assez habile; on trouve une tête encore mieux dessinée à l'intérieur de l'un des cahiers. Enfin, on y voit une petite maison à toit incliné avec deux cheminées, trois fenêtres et une porte sur le côté, une maison française ou québécoise typique. Belmont a-t-il fait ces dessins dans sa jeunesse ou sont-ils l'œuvre d'un écolier de Montréal qui s'ennuyait? Le volume renferme les quatre recueils imprimés suivants, dans l'ordre de la reliure :

(1) Motets pour les principales festes de l'année. A une Voix seule avec la Basse-Continue \& plusieurs petites Ritournelles pour l'Orgue ou les Violles. Pour les Dames religieuses et toutes autres personnes. Par

3 Gallat-Morin, Un manuscrit de musique française classique, 63.

4 Architecte, médecin et musicien accompli, Vachon de Belmont possédait plusieurs livres de musique dont six opéras de Lully et l'Institution harmonique de Salomon de Caus, tous conservés à la Bibliothèque nationale du Québec à Montréal. Le premier orgue de Notre-Dame de Montréal (avant 1705), don d'un Sulpicien, est probablement le sien (voir Gallat-Morin, Jean Girard, musicien en Nouvelle-France, 240-43). Fils d'une famille de hauts fonctionnaires, il n'a pas hésité pas à employer sa fortune qui était considérable pour la construction de l'église et de l'école. De sa première mission qu'il a construite au Fort de la Montagne, il subsiste encore deux tours que l'on peut voir de la rue Sherbrooke, sur la propriété du Grand Séminaire, entre les rues Guy et Atwater.

50 a réservé le même sort aux déesses trop décolletées dans les gravures illustrant les opéras de Lully qui appartenaient aussi à Vachon de Belmont (BNQ, collections spéciales). Cela reflete la pudeur des milieux ecclésiastiques à la fin du XVII ${ }^{e}$ siècle. 
M. NOEL, Maistre de Musique (Paris : Christophe Ballard, 1687), [4]. 70, [2] p.

(2) Second livre d'Airs spirituels de differents autheurs a deux parties (Paris : Christophe Ballard, 1679), [2], $16 \mathrm{f}$.

(3) Premier livre d'Airs spirituels de differents autheurs a deux parties (Paris : Christophe Ballard, 1679), $19 \mathrm{f}$.

(4) Airs a quatre parties avec la basse-continue et quelques-uns à trois en forme de Motets à la fin du Livre, sur la Paraphrase de quelques Pseaumes \& Cantiques de Messire Anthoine Godeau Evesque de Vence. Composez par Henry Du Mont, Organiste de la Reyne \& de l'Eglise S. Paul, Dessus, et basse-continue (Paris : Robert Ballard, 1663), [2], 34, [2] f.

Les titres de ces recueils ne sont pas sans soulever des interrogations quant à leurs compositeurs. Si Henry Du Mont nous est connu par ses messes dites « royales », nous ne savons rien de ce Monsieur Noël qui a composé les motets à l'intention des Dames religieuses et encore moins des « différents autheurs » des airs spirituels. Nous pouvons également nous demander quel a été l'apport de ce répertoire à la vie musicale de la colonie et dans quelles circonstances il aurait pu être exécuté.

\section{Motets de M. Noël}

Ces motets « pour les principales festes de l'année » ont été composés sur des paroles latines par « $M$. Noël, Maistre de Musique », pour les religieuses du couvent du Val-de-Grâce à Paris « qui attirent les Peuples chez elles par la beauté de leurs voix, aussi bien que par la magnificence Royale de leur Église et de leur Maison ", dit l'Avis placé au début du recueil. En effet, la reine Anne d'Autriche avait conservé un appartement dans ce couvent où elle pouvait se retirer à l'occasion. Les motets à voix seule avec basse continue, qui paraissent en 1687 chez Ballard à l'intention des dames religieuses « et toutes autres personnes », présentent un choix de 20 chants pour le Saint-Esprit, Noël, la Semaine sainte, l'Ascension, l'Assomption, le Saint-Sacrement, la messe des morts, la Sainte Vierge, saint Benoît et enfin deux versions du Domine salvum fac Regem, hymne en l'honneur du roi que l'on entonnait à la fin des offices.

Le recueil est dédié à "Monsieur Le Bègue, organiste du Roy et de Saint Mederic ». Le texte de la dédicace met en valeur les qualités tant personnelles que musicales du maître, "un homme qui mesle avec tant de soin les devoirs de la Religion à l'habileté pour la Musique. » On y vante son savoir, sa piété, sa charité, ainsi que son humilité. En effet, Nicolas Lebègue (1631-1702) jouissait à.son époque d'un grand prestige, qui s'étendait bien au-delà des frontières de la France : " à la Cour, comme à la Ville, on se fait un plaisir de vous prester l'oreille».

Noël devait être l'un des nombreux élèves de Lebègue qui fréquentaient son domicile rue Simon-le-France. Serait-ce Noël Cognet de Grantin, ou encore le fils de l'organiste de Sainte-Croix en la Cité, Claude Noël? On sait également qu'en 1691, M. Noël, qui habitait lui aussi la rue Simon-le-Franc (chez son 
maître?), vendait les ouvrages de Lebègue ${ }^{6}$. Or, fait étrange, en 1708 , six ans après la mort de Lebègue, les motets bénéficient d'un nouveau tirage. Cette fois, c'est Nicolas Lebègue lui-même qui est donné comme en étant le compositeur. La dédicace-hommage est reprise intégralement; l'Avis est légèrement modifié et abrégé.

Pour quelles raisons Lebègue n'a-t-il pas publié les Motets sous son propre nom dès 1687 ? Sont-ils le fruit d'une collaboration entre lui et ses disciples? L'un d'entre eux, Noël, aurait mené le projet à bien et y aurait ajouté un hommage à son maître. Ou encore, voulait-il livrer de manière anonyme sa première publication de musique vocale?

Toujours est-il que ces belles pièces sont tombées dans l'oubli. En effet, seuls deux exemplaires de la première édition de 1687 ont survécu : en Allemagne, dans la bibliothèque du comte von Schönborn-Wiesentheid, et aux États-Unis, à la Boston Public Library; aucun n'est connu en France ${ }^{7}$. Et voilà qu'est récemment mis au jour un troisième exemplaire, qui a été conservé dans le Nouveau Monde presque depuis le moment de sa publication.

Souvent très expressifs, ces motets de Noël/Lebègue, avec leur ornementation et leurs vocalises parfois élaborées, requièrent sans aucun doute une certaine habileté de la part de l'exécutant. Ces pièces offrent en outre la particularité de renfermer " plusieurs petites Ritournelles pour l'Orgue ou les Violles » afin de permettre «à la personne qui chante le loisir de se délasser». Ces ritournelles apparaissent dans tous les motets sauf sept; elles sont avant tout destinées à l'orgue puisqu'on va jusqu'à donner des indications de registration : «Grands jeux » ou « Jeux doux ». L'orgue peut intervenir en soliste jusqu'à quatre fois à l'intérieur d'une pièce et ce pour une durée de trois à douze mesures; souvent il annonce un motif à venir, ou il reprend celui qui vient d'être chanté.

On voit que le livre a servi. En effet, plusieurs pièces comportent des annotations ou des ajouts comme dans le tout premier Motet pour le S. Esprit, Veni sancte Spiritus, au début duquel sont marqués le ton de " $\mathrm{g}$ re sol b mol » et la métrique « 2 t. ». Dans le courant de la pièce, on trouve les indications « med. » et « dom. » pour les cadences, ainsi que le mot « fin » pour le cas où l'on aurait besoin d'une version plus courte. Dans ce motet, qui de toute évidence a été chanté, l'orgue se fait entendre d'abord sur les Grands jeux pendant sept mesures, puis plus loin sur le Jeu doux pour trois mesures, avec une dernière ritournelle de quatre mesures, de nouveau sur les Grands jeux,

6Pour une étude du recueil et notamment de l'entourage de Lebègue, voir Norbert Dufourcq, "Autour de Nicolas Lebègue : un recueil de motets inédits ", Recherches sur la musique française classique 25 (1987) : 7-26.

7RISM : 1687, D-brd WD, US Bp; 1708, D-brd WD. Ce n'est qu'en 1987 que le regretté Norbert Dufourcq, lointain successeur de Lebègue à l'orgue de Saint-Merry, a publié un article sur les motets. Plus récemment a paru une édition en fac-similé des motets (Courlay, France : Fuseau, 1994). Le Studio de musique ancienne de Montréal vient d'enregistrer deux d'entre eux, Salve regina et Regina cali, pour la toute première fois, semble-t-il : Le chant de la Jérusalem des terres froides : Québec, Montréal, Indiens Abénakis, coll. Les Chemins du baroque, Nouvelle-France; Wanda Procychin, soprano, Réjean Poirier, orgue; K617 [nom de l'étiquette] K617052 M7 876 DD (1995), 23-24. 
près de la fin de la pièce. Enfin, notons qu'en bas de la dernière page, Jean Girard a écrit : Surge qui dormi, et exsurge a mortuis.

L'organiste et chantre ajoute même deux pièces de sa main. Il recopie le Panis angelicus de la page 28 dans des portées laissées libres dans le bas de la page d'en face. Il s'agit en fait d'une adaptation qui simplifie et raccourcit le motet de Lebègue. Girard se limite aux deux premières sections de l'hymne et, de 24 mesures, en ramène la durée à 21 (la pièce entière fait 40 mesures); il supprime des vocalises en les remplaçant par des valeurs longues et, pour terminer la pièce, il emprunte les trois dernières mesures de la toute fin du motet de Lebègue.

Sur les deux portées libres qui suivent le Panis angelicus imprimé, à la page 31 , on a noté, d'une autre main semble-t-il, une pièce très simple à trois temps, sans paroles. Puis, sur les deux dernières portées, Girard a transcrit l'hymne Maria mater gratia mater misericordia. En bas de la même page, on peut lire l'inscription « Monsieur Bellemont Grand Viquere ${ }^{8}$ dus ... »; la suite a disparu lorsqu'on a coupé le bas de la page.

\section{Airs spirituels de « différents autheurs »}

Les deux recueils suivants portent le titre de "Airs spirituels de differents autheurs a deux parties »; ils ont été publiés, en caractère d'imprimerie, chez Christophe Ballard à Paris en 1679. La fin du XVII ${ }^{e}$ siècle et le début du XVIII siècle ont vu la publication de nombreux recueils de ce genre. Mais, vérification faite dans les divers fichiers et répertoires, il n'y a aucune trace d'un ouvrage portant exactement ce titre; il n'y a non plus aucun exemplaire connu, dans les collections parisiennes du moins. Il restait à tenter d'identifier le contenu de ces recueils, ainsi que les compositeurs des pièces. Le Second livre, le premier dans l'ordre de la reliure et celui qui contient la préface, est composé de 26 cantiques en français sur des paroles édifiantes; le Premier livre en contient 22. L'avis «Au lecteur » indique que ces airs spirituels, toujours qualifiés "de differents Autheurs », bénéficient dans l'édition présente d'une meilleur présentation que cela n'a été le cas auparavant. Diverses améliorations ont été apportées : lettre capitale au début des vers, ponctuation, "paroles mises justement sous les notes où elles doivent estre ", reprises et répétition indiquées, la version précédente étant qualifiée de "presque pas chantables ».

Ballard indique qu'il a mis les ornements ordinaires, car, dit-il, « tous ceux qui apprennent la Musique [...] se forment assez facilement à cette belle maniere de chanter, par les soins des habiles Maistres qui leur montrent. » Il ajoute que « le nombre dans ce bel Art est à present si grand, que ce seroit une presomption ridicule de s'estimer un Phœnix dans la belle maniere de chanter; \& l'unique capable de la bien montrer. » Il ne nomme pas la personne visée.

Contrairement à ce que laisse entendre le titre, il s'avère que les airs spirituels publiés par Ballard ne sont pas du tout de différents auteurs; il s'agit

8Le Supérieur des Sulpiciens était effectivement à Montréal le vicaire général de l'Évêque qui avait son siège dans la ville de Québec et dont le diocèse s'étendait à toute la Nouvelle-France. 
entièrement des airs composés par Bénigne de Bacilly9 (v. 1625-90) « sur les stances chrestiennes de Monsieur l'Abbé Testu », qu'il avait fait graver dès 1672 par G. de Luyne; Bacilly fera paraître une autre édition cinq ans plus tard, suivie d'une édition augmentée en $1683^{10}$.

Aujourd'hui, le nom de Bacilly nous est connu principalement pour sa méthode de chant. Il est évident par l'avis qui précède ses airs qu'il considère comme incontournable son Traité de la méthode de chanter, "lequel sans doute peut donner de grandes lumieres a ceux qui aspirent à la perfection du chant »; il fustige "l'ignorance ou [...] la malice des maîtres », particulièrement ceux qui prétendent " que le chant ne s'apprend point par les Livres. » Ballard s'en prenait à cette exclusive.

En 1688, Bacilly riposte en faisant graver ses Airs spirituels « dans un plus grand nombre et une plus grande perfection que dans les precedentes editions. " Il se réfère à l'édition qui en a paru " en caractères ordinaires de musique » (celle de Ballard, qui détenait le monopole de l'imprimerie de la musique) en avouant qu'il aurait pu intenter un procès à ce dernier pour avoir imprimé ses airs avant l'expiration de son privilège. Néanmoins, Bacilly préfère être grand seigneur et écouter ceux qui considèrent qu'il tire gloire du risque que l'imprimeur a bien voulu prendre. Après la mort de Bacilly, Ballard lui rend enfin son dû : paraissent chez lui en 1692 les deux livres des Airs spirituels de feu M. de Basilly, suivis d'une nouvelle édition du Premier livre en 1693 et du Second livre en $1703^{11}$. C'est donc une édition pirate des Airs spirituels de Bacilly que possédait François Vachon de Belmont! Il devait souvent accompagner ces airs sur son luth, avant de remettre le recueil à son jeune collègue fraîchement débarqué en Nouvelle-France.

L'imprimé ne comporte que peu de chiffrages à la basse pour aider celui qui doit réaliser un accompagnement. C'est pourquoi, dans presque toutes les pièces, un chiffrage très détaillé a été ajouté à l'encre dans 23 pages sur 31 du Second livre et dans 4 pages seulement du Premier livre. Il est difficile de savoir si le chiffrage est de la main de Belmont ou de Girard. Autre ajout manuscrit, au tout début du Second livre, on a marqué le nom des lignes et des

\footnotetext{
9 Nos remerciements vont à $\mathrm{M}^{\mathrm{me}}$ Bénédicte Mariolle, de l'Atelier d'études sur la musique française des XVII ${ }^{e}$ et XVIII ${ }^{e}$ siècles du Centre de musique baroque de Versailles, pour son aimable collaboration et particulièrement à MM. Laurent Guillo et Denis Herlin, musicologues, qui nous ont suggéré d'examiner les Airs de Bacilly.

10Cette dernière édition est signalée par la regrétée Denise Launay, qui attire l'attention sur la complexité des éditions successives de Bacilly dans La musique religieuse en France du Concile de Trente à 1804 (Paris : Société française de musicologie/Klincksieck, 1993), 375, 372 n.153. Voir aussi Gallat-Morin, « Reperes bibliographiques : les airs spirituels de Bacilly et les airs spirituels de différents auteurs publiés à Paris chez Ballard (1679), et conservés à la Bibliothèque nationale du Québec ", Bulletin de l'Atelier d'études sur la musique française des XVIT \& XVIIT siècles, Centre de musique baroque de Versailles, $n^{0} 5$ (1994-95) : 8-9.

11 Le contenu de toutes les éditions de Ballard est le même, mis à part le mot « Mondains » dans un titre chez les « différents auteurs » de 1679 qui devient « Mortels » dans les éditions posthumes qui portent le nom de Bacilly, tout comme dans l'édition gravée de ce dernier de 1688. Par ailleurs, la distribution des airs entre les deux livres est différente dans les éditions Ballard de l'édition améliorée de Bacilly de 1688, auquel ce dernier a aussi ajouté des airs.
} 
interlignes en face de la portée. L'écriture est si petite qu'on ne reconnaît pas bien la graphie; toutefois ce pourrait être plutôt la main de Belmont.

\section{Airs d'Henry Du Mont}

Henry Du Mont (1610-84) est connu pour ses Messes royales en plain-chant musical qui ont été chantées, tant en France qu'au Québec, jusqu' au milieu du $\mathrm{XX}^{\mathrm{e}}$ siècle. Né près de Liège, Henry de Thier avait francisé son nom en Du Mont et fait carrière à la Chapelle royale de Versailles et à Paris. Il avait déjà publié des Cantica sacra (1652) lorsqu'en 1663, à l'instar de plusieurs compositeurs avant lui, il met en musique à son tour les fameuses paraphrases de psaumes publiées en 1648 par Antoine Godeau, évêque de Grasse et de Vence. « Ce poète de ruelles, converti, puis promu à l'épiscopat », comme le décrit Denise Launay, espère « tirer les catholiques de leur mutisme et de leur tiédeur, en imitant les protestants $»^{12}$ qui sont nombreux dans son diocèse. À cette fin, Godeau incite "quelqu'un de ces excellens musiciens qui honorent notre siècle à donner des Airs agréables \& faciles, afin de les rendre populaires. ${ }^{13} \mathrm{Du}$ Mont répond à ce souci en expliquant dans son "Advertissement » qu'il s'est « estudié à chercher la facilité du Chant pour toutes sortes de personnes. "

Dans ses Airs à quatre parties, Du Mont met en musique, en premier lieu, 30 psaumes à 4 parties avec basse-continue. Suivent 10 Airs a trois voix \& basse-continue en forme de motets, toujours en français, mais bénéficiant d'un traîtement musical plus élaboré. Il ajoute à ces dernières pièces une quatrième partie pour « un Dessus de Viole, ou Violon, de laquelle on se servira si l'on veut. » Trois pièces latines terminent la collection, dont une Antienne de Sainte Cecille Benedico te. Viennent enfin un Laudate Dominum omnes gentes et un Domine salvum fac Regem. Pour James R. Anthony, les airs de Du Mont « sont français dans l'âme [...] avec leur coupe binaire, leurs phrases asymétriques, leurs changements de mètre intermittents et leur traitement syllabique du texte ${ }^{14}$. »

Du Mont a « mis la Basse-Continue avec le Dessus, pour la commodité de ceux qui voudront joüer des Instruments, $\&$ chanter ensemble. » Cette version, qui a bénéficié d'un tirage plus important ( 500 exemplaires) pour être vendue séparément, est celle que possédait François Vachon de Belmont. On n'en connaît que six exemplaires dans le monde, dont trois sont intégrés à des Recueils d'airs spirituels publiés publiés par Ballard en 1699 et en 1728. Un seul exemplaire de la version complète a survécu' ${ }^{15}$.

12Launay, La musique religieuse, 324-25.

13 Ibid., 325, citant la préface de Godeau.

14James R. Anthony, La musique en France à l'époque baroque (Paris : Flammarion, 1981), 227.

15 Nous sommes redevable à $M$. Laurent Guillo qui nous a aimablement communiqué ces renseignements : l'exemplaire complet est conservé à Cambridge (MC : PL 1644-1647); le dessus avec basse-continue se trouvent à Montréal et à Oxford (CCL : Mus. 263), à Paris (BNF (Mus.) : Rés 1653 et Vm1 1566) (Recueil de 1699); le Recueil de 1728 est dans les collections de Krakow (BC : 2994 ew.1.) et de Londres (BL : K.7.c.8). Une partie de haute-contre existe à Lyon (BM : Rés FM 133986) et deux exemplaires du Bas-dessus sont à Paris (BNF (Mus.) : Rés. 1653 et Vm1 1566). 
Dans l'exemplaire conservé à Montréal, à la fin des deux psaumes qui se suivent - « VII. Quand l'esprit accablé (Cum invocarem) » et « VIII. Seigneur qui connois (Salvum me fac Domine) »-, on a tracé à l'encre des portées de cinq lignes. Sur la quatrième ligne de chacune des portées est placée une clé de $f a$ dessinée comme dans le plain-chant. Des barres verticales, semblables à des barres de mesure, séparent les groupes de notes en rondes, chaque section étant coiffée de la mention « $1^{\text {ere }}$ corde », " $2^{\text {de }}$ corde », etc., ainsi :

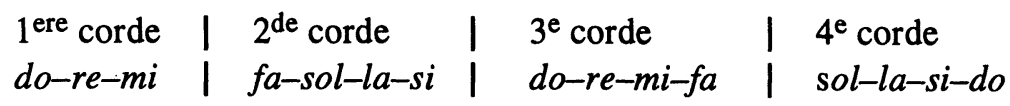

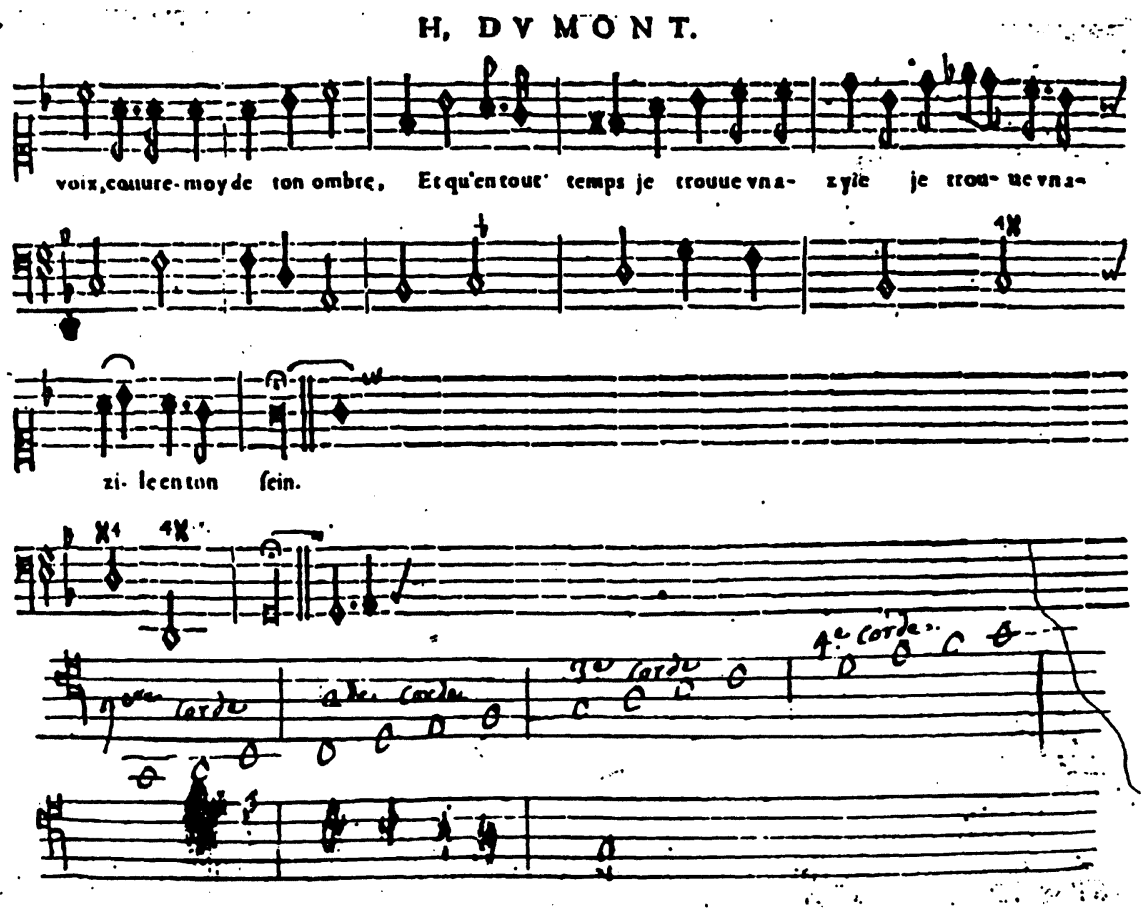

Figure 1 : Gamme de do pour basse de violon. Ajout manuscrit à la p. 6 des Airs à quatre parties d'Henri Du Mont (1663). Montréal : Bibliothèque nationale du Québec, Division des collections spéciales, RES AF 264.

De prime abord, on croit être en présence du système d'accord d'un instrument qui serait accordé do-fa-do-sol, à partir du do situé deux octaves en dessous du do central, ce qui est très bas. Cela ne correspond à aucun des accords des plus courants de la viole, du violoncelle ou de la guitare. Cependant, si l'on fait abstraction des barres, on se rend compte que l'on a affaire tout simplement à une gamme de $d o$ (sur la p. 6 , on a ajouté une deuxième portée avec la gamme de do descendante, dont l'encre est barbouillée) pour 
laquelle on a voulu indiquer sur quelles cordes jouer les notes. Mais de quel instrument s'agit-il? On aura remarqué que la section de la première corde ne contient que trois notes, contrairement aux autres qui en comportent quatre, pour faire commencer la gamme sur $d o$. Si l'on rajoute la note inférieure manquante, si bémol, on obtient un accord par quintes : si bémol-fa-do-sol, qui correspond à l' accord de la basse de violon. En effet, selon Sylvette Milliot, « en 1741, l'accord de notre violoncelle actuel [do-sol-ré-la] vient de s'établir définitivement, remplaçant le précédent, plus bas d'un ton ${ }^{16}$ »[commençant sur le si bémol]. La caisse de la basse de violon est plus longue que celle du violoncelle d'une dizaine de centimètres $(85 \mathrm{~cm}$ contre $75 \mathrm{~cm})$; elle fait partie des bandes de violons, mais on 1'appelle aussi « basse de procession »17. Dans cette fonction, elle est utilisée " pour soutenir les chants et cantiques [...] maintenue au cou de l'instrumentiste par un lien fixé à un trou situé au bas du manche; évidemment, dans cette position, " on ne peut exécuter exécuter ici que des basses très sommaires ${ }^{18}$."

Dans la graphie des mots « lere corde ", etc., on reconnaît la main de Jean Girard, qui aurait ajouté ces portées pour montrer à quelqu'un comment jouer sur la basse de violon. Peut-on en conclure qu'il y avait un tel instrument à Montréal pendant que Girard y habitait, c'est-à-dire entre 1724 et 1765, et qu'il aurait servi à accompagner des airs spirituels, peut-être même dans processions?

\section{Signification de cette collection}

Il est frappant de constater que les quatre volumes reliés ensemble dans le recueil ayant appartenu aux Sulpiciens de Montréal, Belmont et Girard, sont tous des livres rares, voire uniques. Les Motets de Noël/Lebègue est l'un des trois exemplaires de l'édition de 1687 qui ait survécu dans le monde; aucun exemplaire n'est connu en France, pourtant le pays de publication. Les deux recueils d'Airs spirituels de « differents autheurs » semblent être les uniques exemplaires connus de cette édition pirate des airs de Bacilly. Pour leur part, les airs de Du Mont sont moins rares : il existe cinq autres exemplaires connus du dessus avec la basse-continue, mais seulement un exemplaire de la version complète.

Comme les Jésuites dont le Collège à Québec possédaient les exemplaires maintenant uniques de messes de Valentin de Bournonville et d'Arthus-auxCousteaux, actuellement dans les collections du Séminaire de Québec, les Sulpiciens aussi sont responsables de la conservation d'éditions musicales devenues rares en France, quand elles n'ont pas été tout à fait perdues.

Non seulement la mise au jour de ces quatre recueils nous renseigne-t-elle davantage sur le répertoire dont disposait le premier musicien de métier que Montréal ait connu, elle apporte la solution à un problème qui n'était pas totalement réglé. Les deux hymnes manuscrits ajoutés parmi les motets de

16Sylvette Milliot, Le violoncelle en France au XVIII siècle (Paris et Genève : Champion/Slatkine, 1988), 678. Nos remerciements vont à M. Paul-André Dubois, violiste, de nous avoir indiqué cette piste.

17 Ibid., 24 bis.

18Ibid., 39, 679. 
Lebègue ont été fort utiles, en effet, pour identifier de manière définitive la graphie de Jean Girard dont nous avions déjà la quasi-certitude. Il n'y a aucun doute que la main qui a fait les ajouts est la même que celle qui a transcrit un recueil d'hymnes conservé dans les Archives sulpiciennes, ainsi qu'un recueil de cantiques français conservé à la Bibliothèque nationale du Québec à Montréal. Nous avions comparé ces recueils, qui ne comportent aucune signature, au seul texte que nous possédons écrit et signé par Girard : un monitoire qu'il avait recopié en sa qualité de secrétaire de son supérieur, le vicaire général ${ }^{19}$. La comparaison n'était pas convaincante d'emblée en raison d'une différence dans la dimension des caractères et dans la nature de l'écriture, d'une part très appliquée et officielle, d'autre part cursive; néanmoins, après une étude détaillée des textes et plusieurs consultations, nous en étions arrivée à la conclusion que les recueils d'hymnes et de cantiques étaient bien de la main de Girard, ainsi que d'autres documents non signés. Enfin, voici que cette même écriture se retrouve dans un livre de musique que possédait Girard; comme l'écriture n'est pas celle de Belmont, l'autre propriétaire du livre, on y trouve la confirmation que c'est bien la graphie de Jean Girard. Par conséquent, il n'était pas erroné d'affirmer que l'ecclésiastique avait bel et bien recopié environ 300 feuilles de plain-chant pour répondre aux besoins de la communauté montréaliste, comme on disait à l'époque.

\section{A-t-on chanté cette musique à Ville-Marie?}

La découverte du recueil de motets et d'airs spirituels ajoute à la connaissance du corpus de musique présent en Nouvelle-France. Est-il possible de savoir dans quelles circonstances cette musique aurait pu être jouée à Montréal (autrefois Ville-Marie), ville que le recueil n'a jamais quittée? Mais, avant de s'interroger sur cette pratique, voyons à quelle occasion on interprétait cette musique en France où elle a été composée.

Le roi Louis XIV, grand mélomane, devait assister à la messe quotidiennement; elle avait lieu dans sa chapelle et prenait la forme d'une messe basse solennelle. Les musiciens étaient encouragés à composer des motets « pour la Messe du Roy, où l'on en chante d'ordinaire trois [motets], un grand, un petit pour l'élévation et un Domine salvum fac Regem ${ }^{20}$; il va sans dire que l'on renouvelait ce répertoire continuellement. Par ailleurs, Guillaume-Gabriel Nivers a composé en 1698 des Motets à voix seule à l'intention des jeunes filles qui fréquentaient la Maison royale de Saint-Louis à Saint-Cyr, fondée par Madame de Maintenon. De nombreux compositeurs ont publié des livres de petits motets destinées à diverses communautés religieuses, tels les motets de Noël/Lebègue. Ces motets convenaient parfaitement à l'élévation de la messe ou aux vêpres, en plus d'alimenter une pieuse récréation. Les airs spirituels ou

19Archives du séminaire de Saint-Sulpice de Montréal, case $B, n^{\circ} 179$, Montréal, Bibliothèque nationale du Québec, collections spéciales, Mss. 40. Des extraits des documents de la main de Girard sont reproduits dans Gallat-Morin, Jean Girard, musicien en Nouvelle-France, 256, 263, 288, 293, 308, et « Un manuscrit de cantiques », 84, 88-89.

20Pierre Perrin, avant propos des Cantica pro Capella Regis (Paris : Ballard, 1665), cité par Anthony, La musique en France, 231. 
cantiques en français, publiés en grand nombre, étaient encore plus accessibles que les motets en latin. Ainsi espérait-on remplacer les airs profanes et frivoles et encourager les catholiques à chanter des airs pieux chez eux, à l'instar des protestants. Il ne faut pas oublier, enfin, les « innombrables processions qui faisaient partie intégrante de la vie religieuse parisienne au XVII ${ }^{\text {. }}$ » Lors de la solennité de la Fête-Dieu, " la procession visitait un certain nombre de reposoirs où était exposé le Saint-Sacrement et à chaque reposoir on chantait un motet ou une partie de motet ${ }^{21}$. » Les mêmes pratiques avaient-elles cours en Nouvelle-France?

Lorsqu'il est missionnaire au Fort de la Montagne, en dehors des limites de Villemarie, François Vachon de Belmont «ne se contente pas [d'apprendre aux garçons indiens] la doctrine chrétienne et la manière de bien vivre, écrit Mgr de Saint-Vallier, évêque de Québec, il leur enseigne aussi à parler le français et à chanter le plain-chant et la musique, selon qu'ils ont de la voix ${ }^{22}$." Belmont a pu accompagner sur son luth les airs contenus dans les recueils de Bacilly et de Du Mont; les motets de Noël/Lebègue, publiés sept ans après son arrivée à Montréal en 1680 , ont dû lui être envoyés par la suite. Comme Belmont se faisait des scrupules de son amour de la musique, le supérieur de Paris a dû le rassurer : "Continuez a dissiper vos tentations et vos ennuys et a divertir vos sauvages et vos frères en iouant de vostre luth ${ }^{23}$. »

Vers la fin du recueil de Du Mont (p. 32v), le nom « De Casson » est inscrit en haut de l'Antienne de Sainte Cécille. Il s'agit du premier supérieur des Sulpiciens de Montréal, mort en 1701, à qui Belmont devait succéder. Quelle est la raison de cette inscription? De Casson a-t-il chanté cette antienne? On ne le sait, mais l'on peut supposer qu'à un moment donné à Ville-Marie on a rendu hommage à la patronne de la musique.

On connaît les livres imprimés de motets de Bernier, de Morin et surtout de Campra conservés à Québec, qui appartenaient à l'évêque Saint-Vallier, au Chapitre de la Cathédrale, à l'Hôpital général, ainsi que les motets recopiés dans les manuscrits des Ursulines et des Augustines de 1'Hôtel-Dieu24; en outre, les annales des diverses communautés relatent des cérémonies au cours desquelles étaient chantés des motets. Si le recueil appartenant à Jean Girard comprend les premiers livres de motets retrouvés à Montréal, plusieurs chroniques de l'époque, néanmoins, font allusion au chant de motets dans cette ville.

Le motet se chante chez les religieuses, notamment chez les Hospitalières de Saint-Joseph à l'Hôtel-Dieu de Montréal, comme nous le verrons plus loin. Pour leur part, les religieuses de la Congrégation de Notre-Dame estiment que

21 Anthony, La musique en France, 245.

$22 \mathrm{M}^{\mathrm{gr}}$ de Saint-Vallier, Éstat présent de l'Église et de la colonie française dans la Nouvelle-France, 1685, cité par Bruno Harel, p.s.s., « Le Domaine du Fort de la Montagne (1666-1860) », Le Grand Séminaire de Montréal de 1840 à 1990 (Montréal : Éditions du Grand séminaire de Montréal, 1990), 20.

23Paris, Archives de Saint-Sulpice, Minutes de la correspondance des supérieurs, ms., lettre de Tronson à de Belmont, 1693, t. I, 52.

24Voir Gallat-Morin et Antoine Bouchard, Témoins de la vie musicale en Nouvelle-France, (Québec : Archives nationales du Québec, 1981). 
le fait de consacrer du temps à l'apprentissage du chant n'est pas conforme à leur vocation première d'éducatrices auprès des habitants. On fait exception lors des grandes fêtes comme l'écrit leur fondatrice, Marguerite Bourgeois : "Quelles ne chantent ny grande messe ny vespres dans leur eglise mais quelque motet aux messes basses et aux jours jugez a propos ${ }^{25}$. » Ce n'est qu'en 1720 qu'elles commencent à chanter des motets en musique, lorsque $\mathrm{M}^{\mathrm{gr}}$ de Saint-Vallier, deuxième évêque de Québec, écrit à la supérieure, Sœur Lemoyne : «Je ne vois pas d'inconvénients de permettre quelque fois à vos sœurs de chanter des motets en musique aux grandes fêtes ${ }^{26}$."

Tout comme en France, l'année était ponctuée de nombreuses fêtes religieuses, autant d'occasions pour la population d'aller en procession lorsque la rigueur du climat le permet. Une procession particulièrement émouvante eut lieu en novembre 1724, peu de temps après l'arrivée de Jean Girard à Montréal, lorsque les religieuses de l'Hôtel-Dieu reprirent possession de leur couvent qui avait été détruit par le feu trois ans auparavant. Les religieuses étant accueillies à la sacristie de l'église paroissiale par le Supérieur des Sulpiciens, François Vachon de Belmont, la procession se forma et se dirigea le long de la rue Saint-Joseph (aujourd'hui la rue Saint-Sulpice) jusqu'à l'Hôtel-Dieu située rue Saint-Paul. Laissons la parole à l'annaliste, la sœur Marie Morin : « On nous donna des cierges du seminere et on nous fit marcher deux a deux devand le tres Saint Sacrement. Tout le clergé y estoit chantant des himnes et motets en musiques avec les instrumans ${ }^{27}$. " L'annaliste ne nous dit pas de quels instruments il s'agit : d'une basse de procession peut-être, entre autres? Elle ajoute que la célébration se termina par « un très beau salut [...] le clergé chanta, et nos sœurs chantres aussy meslerent leurs voix avec celles des prestres et instrumans qui firent une melodie tres agreable qui portèt au Ciel tout vivand. »

Les processions de l'année conduites avec la plus grande solennité étaient les deux processions de la Fête-Dieu, avec arrêt et chant à chaque reposoir : à la Congrégation Notre-Dame, chez les Jésuites et à l'Hôtel-Dieu. « Arrivé à l'Hôtel-Dieu, on ne chante rien qu'après le Motet chanté par les Religieuses » relate le Recueil des usages de la paroisse ${ }^{28}$. Et la sœur Morin de raconter : " on fait de grandes décharges de fusils et mesme de canons », avec le résultat qu'en 1721 « un estourdy tira dans le portail de l'église[, ce qui] porta le feu dans la voutes en un momand ${ }^{29}$. » Par ailleurs, nous apprenons par les archives de la paroisse Notre-Dame de Montréal que, du mois de mars 1729 à mars 1730,

25Montréal, Archives de la Congrégation de Notre-Dame, Écrits autographes de la Bienheureuse Marguerite Bourgeoys (avant 1700), photocopie de la copie faite en 1879 et déposée dans les Archives de l'Archevêché de Montréal, 6.

26Montréal, Archives de la Congrégation de Notre-Dame, Sœur Sainte Henriette, Cahier dactylographié, 1707-1759, Cahier $6^{\mathrm{e}}$ (début $\mathrm{XX}^{\mathrm{e}}$ siècle), 309.

27Marie Morin, Histoire simple et véritable [...], Les Annales de l'Hôtel-Dieu de Montréal, 1659-1725, édition critique par Ghislaine Legendre (Montréal : Les Presses de l'Université de Montréal, 1979), 290-91 (ms. 314-315).

28 Archives du Séminaire de Saint-Sulpice de Montréal, Recueil des usages de la paroisse de Montréal, ms., 1796, 114.

29 Annales de scur Morin, 292, citées par Esther Lefebvre, Marie Morin, premier historien canadien de Villemarie (Montréal : Fides, 1959), 178. 
année du Jubilé, « il y eut quantité de Demoiselles de la Ville à qui on apprenoit des Cantiques Spirituels et des Motets, et qui les chantoient soit au sermon soit au salut ${ }^{30}$. »

Les annotations sur plusieurs pages des quatre recueils d'airs et de motets montrent bien que ces livres ont vraiment servi. Jean Girard a dû jouer un rôle central dans l'organisation de la musique pour les diverses cérémonies, faisant entendre à la population en ces occasions les ouvres des grands compositeurs français. Est-ce lui qui a appris des cantiques spirituels et des motets aux demoiselles de la ville? Il a pu en choisir parmi les chants de Lebègue, de Bacilly et de Du Mont. On a très bien pu chanter lors de la Fête-Dieu le Panis angelicus que Girard a recopié, simplifié et raccourci, motet de Lebègue, organiste du roi, dont des pièces figurent aussi dans le Livre d'orgue de Montréal.

\section{Conclusion}

Les quatre recueils de Lebègue, de Bacilly et de Du Mont découverts à Montréal ajoutent à notre connaissance de la bibliothèque musicale de l'organiste et maître d'école Jean Girard et nous renseignent davantage sur la musique qui a pu être chantée lors des nombreuses cérémonies dont nous parlent les annales. En outre, des annotations manuscrites dans le recueil de Dumont suggèrent la présence à Montréal d'une basse de violon ou «basse de procession », qui viendrait s' ajouter aux instruments déjà connus. D' autre part, nous pouvons constater que non seulement les collections du Québec renferment un corpus intéressant de musique religieuse apportée de France sous l'Ancien régime, mais que sont conservées dans nos archives des œuvres rares sinon uniques, qui représentent une partie du patrimoine musical français qui n'a pas survécu en France même.

\section{Résumé}

Un recueil donné à la Bibliothèque nationale du Québec (Montréal) en 1993 porte les signatures de François Vachon de Belmont, supérieur des Sulpiciens de Montréal de 1701 à 1732, et de Jean Girard, à qui appartenait le Livre d'orgue de Montréal. Les quatre volumes renferment les Motets (1687) de M. Noël (pseudonyme de Lebègue), les Airs spirituels de " differents autheurs 》 (1697), édition " pirate » d'œuvres de Baccilly, et les Airs à quatre parties (1663) de Du Mont. Très rares, voire uniques, ces œuvres complètent la bibliothèque musicale de Jean Girard et ajoutent à notre connaissance du répertoire musical interprété à Ville-Marie au XVIII ${ }^{\mathfrak{e}}$ siècle. 